\title{
Lost in transition
}

$\mathrm{F}$ rom a patient's perspective, cancer is not only a physical illness. The emotional toll incurred upon diagnosis can be equally devastating but is often overlooked. As a cancer survivor, I know that for young adults this emotional hardship could not come at a worse time. Young adult's lives are inherently transitional as we move from the security of adolescence to independently developing careers and families. This rapid development and the accompanying fast-paced lifestyle are stalled by the diagnosis of cancer, creating great discordance.

My diagnosis came just before I was supposed to start university and led me to miss my first semester. Seven years later, I completed treatment and expected to regain my former life, but discovered that re-integrating into a cancer-free life was incredibly difficult. Previous work has shown that the inability to re-integrate can initiate feelings of isolation, anxiety, decreased self-esteem and depression which may be long-lasting without the proper support. ${ }^{1}$ An apparent flaw in our health care system is the lack of emotional support for this post-treatment barrier, forcing young adults to face this alone.

The most difficult psychological aspect of re-integrating into my "old" life was overcoming social isolation. This is one of the most devastating and yet understudied emotional trials for young adult patients with cancer. In my opinion, this trial begins during treatment while navigating a medical system generally designed for a much older patient population. ${ }^{1}$ It was a surreal feeling to walk into a world-renowned cancer centre and be the youngest person there by a minimum of 30 years. In an environment that is already anxiety ridden, this lack of peer-support tailored to a young adult compounds the problem. I find it unacceptable that only five centres in Canada have this level of peersupport . ${ }^{2}$

The isolation continued post-treatment, manifested by the inability to re-

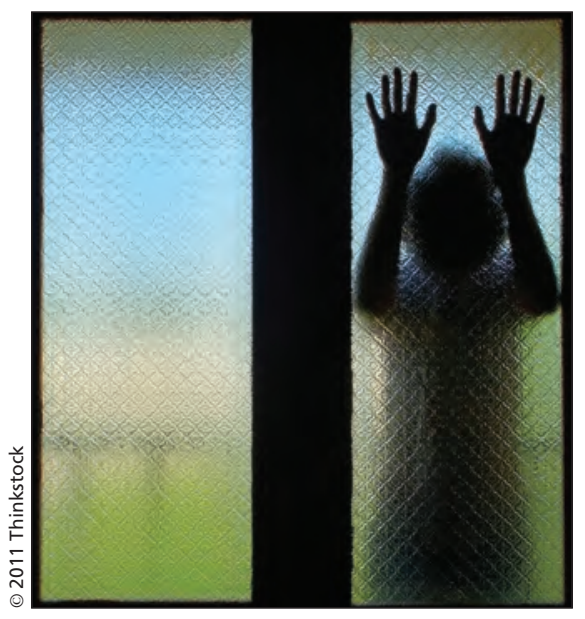

integrate into a cancer-free life. I was segregated by my physical appearance and emotional instability, which made it difficult to converse normally with my peers. This in turn affected my personal relationships and academic endeavors. This post-treatment isolation was compounded by the same lack of peer-support that exacerbated the intreatment isolation.

Although it would seem that isolation would dissipate with time, the emotional long-term effects can influence patients well after treatment has finished. ${ }^{3}$ Since young adults have their entire lives ahead of them, emotional support networks need to be implemented to attenuate long-term emotional damage due to isolation.

Based on the psychosocial challenges young adult patients face and the corresponding long-term effects, it is critical that targeted health care policies meet these patient's post-treatment needs. Upon completing treatment, it was left up to me to go and find the support I needed. Locating the appropriately supportive organizations is a very difficult task for survivors who, after finishing treatment, are fatigued or physically unable to complete this search. In my opinion, this situation is unacceptable.

Post-treatment care support groups tailored to young adults should be a mandatory component of all major cancer centres. A way of supplementing a costly professionally led system is to use a peer-led support system which have been shown to have few qualitative differences. ${ }^{4}$ Helping young adult survivors create these groups, would allow both emotional support and encourage the formation of communities or "cancer families" which could further reduce the feeling of isolation. ${ }^{5}$ Whether professionally led or peer-led, these support groups will create a community for young adults and attenuate feelings of isolation and longterm associated distress.

Only when these support systems are nationally accessible can we effectively help all young adults' psychological needs. By removing the onus of young adults to find their own support systems, their focus can shift from surviving to thriving.

\section{Timothy W. Buckland BSc \\ Department of Biochemistry \\ University of Alberta \\ Edmonton, Alta.}

View a video interview with the author at www.aacr.org/home/public--media /multimedia-/aacr-podcasts/annual-meeting -2011-podcasts-and-teleconferences /interview-with-timothy-buckland.aspx

This article won a competition seeking submissions on the subject of cancer for the Canada-wide student journal, Health Science Inquiry (hsinquiry.sa.utoronto.ca).

\section{References}

1. Rabin C, Simpson N, Morrow K, et al. Behavioral and psychosocial program needs of young adult cancer survivors, Qual Health Res 2010;21:796-806.

2. Canadian Cancer Society's Steering Committee. Canadian cancer statistics 2009. Toronto (ON): The Society; 2009

3. Treadgold CL, Kuperberg A. Been there, done that, wrote the blog: the choices and challenges of supporting adolescents and young adults with cancer, $J$ Clin Oncol 2010;28:4842-9.

4. Stevinson C, Lydon A, Amir Z. Characteristics of professionally-led and peer-led cancer support groups in the United Kingdom, J Cancer Surviv 2010;4: 331-8.

5. Ussher J, Kirsten L, Butow P, et al. What do cancer support groups provide which other supportive relationships do not? The experience of peer support groups for people with cancer, Soc Sci Med 2006;62:2565-76. 